\title{
Erratum: Effect of density and nucleon-nucleon potential on the fusion cross section within the relativistic mean field formalism [Phys. Rev. C 101, 044603 (2020)]
}

\author{
M. Bhuyan, Raj Kumar $\odot$, Shilpa Rana, D. Jain, S. K. Patra, and B. V. Carlson
}

(Received 3 May 2021; published 30 November 2021)

DOI: 10.1103/PhysRevC.104.059901

In the original paper, we have also considered a few reactions with heavier targets, i.e., ${ }^{48} \mathrm{Ca}+{ }^{238} \mathrm{U},{ }^{48} \mathrm{Ca}+{ }^{248} \mathrm{Cm}$, and ${ }^{64} \mathrm{Ni}+{ }^{238} \mathrm{U}$. Also in Fig. 4 of our paper, the experimental data along with the calculated cross sections corresponding to these reactions are of the capture cross section. Hence, the typographical error (s) throughout the paper, i.e., the term "fusion cross section" should be read as "fusion and/or capture cross section."

In Sec. II of the our paper, a general expression for the penetrability $P_{\ell}$ in terms of the barrier height $V_{B}^{\ell}\left(E_{\mathrm{c} . \mathrm{m} .}\right)$ where (c.m.) is the center of mass and curvature $\hbar \omega_{\ell}\left(E_{\mathrm{c} . \mathrm{m} .}\right.$.) of the interaction potential is given in Eq. (12) [1]. Here, it is worth mentioning that for the particular case in the original paper, the $V_{B}$ and $\hbar \omega$ are independent of center-of-mass energy $E_{\mathrm{c} . \mathrm{m} .}$. More details can found in Ref. [1], which is listed as Ref. [74] in the original paper.

However, all our calculations are based on the correct equation(s) together with correct experimental data. In other words, except for the above, the rest of the above article, including all results, remains unchanged.

We are thankful to M. V. Chushnyakova, I. I. Gontchar, and O. M. Sukhareva for bringing the above-said typographical errors to our attention.

[1] R. Kumar, M. Bansal, S. K. Arun, and R. K. Gupta, Phys. Rev. C 80, 034618 (2009). 